\title{
A IMPORTÂNCIA DA TEORIA BIOECOLÓGICA NA COMPREENSÃO E DIAGNÓSTICO DO FENÓMENO DOS MAUS TRATOS INFANTIS
}

\author{
Elisabete Lopes \\ Doutoranda em Estudos da Criança - CIEC, Instituto de Educação, Universidade do Minho, Braga, Portugal \\ id9952@alunos.uminho.pt \\ Zélia Caçador Anastácio \\ CIEC, Instituto de Educação, Universidade do Minho, Braga, Portugal, zeliaf@ie.uminho.pt
}

Recepción Artículo: 13 octubre 2021 Admisión Evaluación: 13 octubre 2021 Informe Evaluador 1: 13 octubre 2021 Informe Evaluador 2: 14 octubre 2021 Aprobación Publicación: 14 octubre 2021

\section{RESUMO}

A Criminologia, ciência empírica e multidisciplinar, tem como objeto de estudo o crime e estuda-o de acordo com a perspetiva biológica, psicológica e social, atendendo a três vertentes, três personagens e três atos. Urie Bronfenbrenner desenvolveu o modelo ecológico e posteriormente adaptou-o para o designado modelo bioecológico do desenvolvimento humano. 0 mesmo tem sido usado por diversas disciplinas para a explicação de fenómenos como a violência. Esta teoria estuda o desenvolvimento humano de acordo com a interação do processopessoa-contexto-tempo, sendo aplicada em meio natural de vida, podendo ser usada para compreender o desenvolvimento infantil e, por conseguinte, o fenómeno dos maus tratos praticados contra crianças, pois permite analisar os fatores de risco e de proteção ao mesmo tempo que averigua as suas relações. 0 presente trabalho apresenta uma breve resenha sobre a teoria bioecológica e a sua aplicabilidade em possíveis situações de maus tratos contra crianças, envolvendo a importância da prevenção e intervenção com grupos de risco, podendo contribuir para um possível diagnóstico destas situações.

Palavras-chave: teoria bioecológica; desenvolvimento infantil; violência; maus tratos contra crianças

\section{ABSTRACT}

The importance of Bioecological Theory in the understanding and diagnosis the phenomenon of child maltreatment. Criminology, an empirical and multidisciplinar science, has crime as it s object of study and studies it according to the biological, psychological and social perspective, taking into account three aspects, three characters and three acts. Urie Bronfenbrenner developed the ecological model and later adapted it to the so-called bioecological model of human development. The same has been used by several disciplines to explain phenomena such as violence. This theory studies the human development according to the interaction of 


\section{A IMPORTÂNCIA DA TEORIA BIOECOLÓGICA NA COMPREENSÃO E DIAGNÓSTICO dO FENÓMENO dOS MAUS TRATOS INFANTIS}

the process-person-context-time, being applied in a natural way of life, and can be used to understand child development and, consequently, the phenomenon of maltreatment against children, as it allows to analyze risk and protection factors while investigating their relationships. This paper presents a brief review of the bioecological theory and its applicability to possible situations of maltreatment against children, involving the importance of prevention and intervention with groups at risk, and may contribute to a possible diagnosis of these situations.

Keywords: bioecological theory; child development; violence; child maltreatment

\section{INTRODUÇÃO}

A criminologia, ciência de visão empirista, consciente e multidisciplinar tem como seu objeto de estudo 0 crime e estuda-0 através da identificação de: a) três vertentes - as normas penais definidas, as normas violadas e a reação social; $b$ ) três personagens - os agentes de controlo, a vítima e 0 agressor; e c) três etapas - a prevenção, o ilícito e a resposta penal (Cusson, 2011). Um dos pressupostos da ciência criminológica assenta, como anteriormente mencionado, na prevenção, a qual faz parte da intervenção desenvolvida para a redução da reincidência criminal e se encontra dividida em três níveis: 1) prevenção primária - trata-se da intervenção desenvolvida antes de 0 crime ocorrer; 2) prevenção secundária - destina-se a grupos de risco que apresentam condutas inadequadas que podem levar à prática criminal e 3) prevenção terciária - destina-se aos transgressores identificados que já praticaram atos puníveis por lei (Gendreau \& Andrews cit in Fonseca, Pinho, Simões \& Simões, 2006). Contudo, importa referir que a prevenção do fenómeno criminal se faz atendendo a que "desta causalidade circular resulta um todo que constitui um sistema: se uma parte do todo mudar, o resto tenderá também a mudar" (Cusson, 2011, p.29) e a isto se chama prevenção. Saliente-se que a "(...) criminologia atual dedica-se (...) agora a revelar sobretudo a lógica interna do fenómeno criminal (...) concentra a atenção sobre a natureza íntima do fenómeno e sobre a sua causalidade intrínseca" (Cusson, 2011, p.29). Na ótica da multidisciplinariedade do saber-saber, saber-fazer e saber-ser e para que o estudo do fenómeno criminal seja o mais fiel possível, esta ciência apoia-se na perspetiva tridimensional do Ser Humano, isto é, a correlação do ser bio-psico-social. Como tal a teoria de Urie Bronfenbrenner (Ano) ganha uma importância acrescida para a compreensão do desenvolvimento humano e a interligação com os fenómenos da violência. Posto isto, o presente artigo propõe a conjetura do estudo da problemática dos maus tratos a crianças, sob a perspetiva da teoria bioecológica aplicada ao desenvolvimento infantil, na medida da promoção de saúde e prevenção de possíveis situações de abuso.

\section{BREVE DEFINIÇÃO DE MAUS TRATOS INFANTIS}

A situação de maus tratos infantis ou maus tratos contra crianças "(...) é um grave problema (...) que atinge e prejudica esta população durante importante período de desenvolvimento" (Pires \& Miyazaki, 2005, p. 44). A Sociedade Brasileira de Pediatria (cit in Pires \& Miyazaki, 2005, p. 44), argumenta que a violência exercida sobre crianças acontece quando "um sujeito em condições de superioridade (idade, força, posição social ou económica, inteligência, autoridade) comete um ato ou omissão capaz de causar dano físico, psicológico ou sexual, contrariamente à vontade da vítima, ou por consentimento obtido a partir de indução ou sedução enganosa", o que por si só implica que esta prática, reiterada ou não, seja infligida por alguém mais velho do que a criança, podendo ser um adolescente ou um adulto que preencha estes requisitos. Segundo a APAV (2011) esta tipologia criminal é definida por qualquer tipo de ato que envolva uma ação ou omissão praticada por pessoas próximas da criança ou que tenham com ela uma relação estabelecida por ordem familiar ou profissional. A mesma fonte continua referindo que "qualquer tipo de mau trato (...) atenta de forma direta, contra a satisfação adequada dos direitos e das necessidades fundamentais das crianças e dos jovens, não garantindo, por este meio, o desenvolvimento pleno e integral de todas as suas competências físicas, cognitivas, psicológicas e sócio emocionais" (APAV, 2011, p.12). A mesma ideia é repetida em outras obras (Referências, pelo menos uma para exemplificar) o que denota a existência de uma linha muito ténue que separa a correta satisfação das necessidades de uma situação de risco e/ou perigo. 


\section{A TEORIA DE URIE BRONFENBRENNER - BREVE RESENHA SOBRE 0 MODELO ECOLÓGICO}

Urie Bronfenbrenner desenvolveu entre o Século XX e XXI estudos que serviram de apoio a diversas disciplinas, tendo sido o suporte para a compreensão de acontecimentos como por exemplo a violência (Barreto, 2016). Numa fase inicial os seus estudos estavam focados no desenvolvimento familiar, na perceção de como a mãe afeta o bebé (Barreto, 2016) e de como a criança ao longo da sua vida vai interagindo com diversas pessoas e objetos (Bhering \& Sarkis, 2009). Estes estudos ajudaram na "(...) compreensão sobre como as crianças aprendem e se desenvolvem (...)" (Bhering \& Sarkis, 2009, p. 7). É nesta área do saber que a teoria proposta ganha dimensão e define-se como modelo ecológico. Neste sentido, e segundo as palavras do autor, "(...) a Ecologia do Desenvolvimento Humano é definida como o estudo científico da progressiva acomodação mútua, durante todo 0 curso de vida, entre um ser ativo em crescimento e as propriedades em mudança nos contextos imediatos nos quais a pessoa em desenvolvimento vive (...) nesse processo ela é afetada pelas relações entre esse contexto imediato e os distantes, estando todos estes contextos encaixados" (Bronfenbrenner cit in Barreto, 2016, p. 283). Este modelo possibilita que a atenção seja dividida para a pessoa e ambiente direto, tendo em conta a relação com outros ambientes indiretos (Polleto \& Koller, 2008) que podem influenciar o seu desenvolvimento.

Ao longo do tempo, de vários estudos realizados e através de críticas ao seu trabalho, Bronfenbrenner apercebeu-se das falhas existentes nos seus constructos e propôs a teoria bioecológica para compreender 0 desenvolvimento humano (Barreto, 2016). Este modelo também incorpora 0 meio ambiente, mas sugere que 0 objeto de estudo seja também analisado através de outros componentes (Polleto \& Koller, 2008).

\section{A TEORIA DE URIE BRONFENBRENNER - O MODELO BIOECOLÓGICO}

Como refere Barreto (2016) o avanço do termo, ecológico para bioecológico, deve-se ao facto de Urie Bronfenbrenner reconhecer as lacunas na sua teoria, pois a relação estrutural e funcional (aspetos biológicos, cognitivos, emocionais e comportamentais) do Ser Humano só passou a estar incluída neste modelo, sendo 0 mesmo entendido como a terceira fase de evolução (Rosa \& Tufge, 2013). A derivação de conjeturas singulares explica-se por uma descrição mais completa e sistemática do modelo bioecológico como um todo (Bronfenbrenner \& Ceci, 1994). 0 modelo prevê características distintivas baseadas na apreciação de estruturas denominadas processos proximais que são enquadradas num esquema conceptual que estabelece a variação sistemática em função do conjunto dos processos e do ambiente em que estes ocorrem, do mesmo modo que expõe a variação da transmissão genética em função da caráter dos efeitos do progresso em causa e por último aprecia e define 0 nível completo de atividade do progresso em torno das disparidades pessoais de base genética (Bronfenbrenner \& Ceci, 1994). Este modelo também aborda "(...) o que é provavelmente a limitação mais séria e problemática do paradigma genético comportamental (...)" (Bronfenbrenner \& Ceci, 1994, p. 570). Os mesmos autores sugerem que a pesquisa dos processos proximais e as suas deduções (em virtude do desenvolvimento no mesmo ambiente) consagra um subterfúgio, que pode ser usado para atestar as balizas genéticas e ambientais, cooperando para as divergências singulares ao nível psicológico (Bronfenbrenner \& Ceci, 1994).

Esta teoria centra-se na dinâmica de interrelações (Coscioni, et al., 2018), sendo o desenvolvimento estudado em virtude do resultado da interação Processo - Pessoa - Contexto - Tempo, definido como o modelo PPCT (Bhering \& Sarkis, 2009; Cosconi et al., 2018) de onde se pressupõe que os elementos constitutivos deste influenciam 0 desenvolvimento humano e consequentemente o seu resultado (Rosa \& Tudge, 2013).

\section{Processo}

É percebido como fundamental no desenvolvimento, pois compreende as experiências e significados atribuídos ao meio ambiente no decurso da vida, ocorrendo por desempenho de papéis ou atividades (Poletto \& Koller, 2008; Antoni \& Koller, 2010; Bhering \& Sarkis, 2009). 0 desenvolvimento acontece através da interação entre 0 sujeito e as pessoas presentes no seu meio imediato (Bronfenbrenner \& Morris cit in Collodel Benetti et al. 2013). É definido como um processo proximal - força motora do desenvolvimento - e é compreendido como o papel 


\section{A IMPORTÂNCIA DA TEORIA BIOECOLÓGICA NA COMPREENSÃO E DIAGNÓSTICO dO FENÓMENO dOS MAUS TRATOS INFANTIS}

de cada um no seu processo individual de crescimento, variando consoante as características individuais de contexto e de tempo (Rosa \& Tudge, 2013; Coscioni, et al. 2018; Collodel Benetti et al. 2013). A título de exemplo, "(...) brincar com uma criança, brincar individualmente ou em grupo, atividades entre crianças (...) são engrenagens ao desenvolvimento" (Collodel Benetti et al. 2013, p.92). Bronfenbrenner refere que devem coexistir cinco aspetos para que o processo se estabeleça, nomeadamente: 1) 0 indivíduo deve estar a realizar alguma ação; 2) a ação deve decorrer num determinado período de tempo e com regularidade; 3) a ação deve ir aumentando de complexidade; 4) as relações estabelecidas através destes processos devem ser recíprocas; e 5) os materiais que compõem 0 ambiente onde estes processos se manifestam devem estimular a cognição (Bronfenbrenner cit in Coscioni et al. 2018), defendendo 0 autor que "(...) é através do engajamento nessas tarefas e interações que 0 indivíduo torna-se capaz de dar sentido ao seu mundo, e a partir disso, transformá-lo" (Bronfenbrenner cit in_Collodel Benetti et al. 2013, p.92). É de salientar que estes processos se vão tornando tão mais poderosos quanto mais forte for a relação emocional entre os indivíduos (Cosciono et al. 2018). Esta análise deve ser feita de forma bidirecional, isto é, analisar tanto os elementos objetivos como subjetivos com base na compreensão dos indivíduos (Rosa \& Tudge, 2013; Coscioni et. al, 2018).

\section{Pessoa}

Denominada como eu ecológico e representa o ser humano como um ser biológico, sociológico e psicológico em evolução, onde cada uma destas características interagem entre si produzindo e concebendo resultados (Poletto \& Koller, 2008; Antoni \& Koller, 2010; Bhering \& Sarkis, 2009). Urie Bronfenbrenner descreveu três tipos de características pessoais, entendidas como resultados e como moderadoras, que fazem parte deste elemento, que são: 1) características de força, que podem ser motivadoras ou disruptivas, sendo as primeiras as que sustentam os processos proximais, porque têm como alicerces a curiosidade e 0 incentivo de iniciar atividades/ações, e as segundas que fazem alusão ao elemento impeditivo, o qual está correlacionado com a impulsividade. A título de exemplo, veja-se duas crianças que têm os mesmos recursos. Portanto, o que vai variar será 0 conjunto dos estímulos a receber. Se as crianças forem estimuladas e motivadas terão mais sucesso do que outras crianças que não receberam qualquer estímulo ou este era deficitário; 2) características de recurso fazem alusão a aspetos físicos e materiais, cognitivos, emocionais e sociais da pessoa, isto é, às suas capacidades e necessidades que podem colocar em causa os processos proximais, podendo chegar mesmo a inibi-los, como por exemplo habilidades e inteligência funcionam como características que favorecem os processos, enquanto deficiências genéticas e lesões graves podem perturbá-los; 3) características de demanda representam características que podem causar reações favoráveis ou desfavoráveis, isto é facilitar ou dificultar, reações sociais como por exemplo a cor de pele, influenciando os processos proximais e o desenvolvimento futuro (Barreto, 2016; Coscioni, et. al, 2018; Rosa \& Tufge, 2013; Collodel Benetti et al. 2013).

\section{Contexto}

É descrito como qualquer acontecimento que ocorre fora do organismo e que pode influir ou ser influenciado por quem se encontra em desenvolvimento (Collodel Benetti et al., 2013). É um elemento dividido em cinco níveis e analisado em função destes que são definidos em: 1) microssistema, 2) mesossistema, 3) exossistema, 4) macrossistema e 5) cronossistema os quais dependendo da forma da sua disposição auxiliam na análise de contextos de vida (Collodel Benetti et al., 2013; Coscioni el. al, 2018). No primeiro - microssistema - advêm os processos proximais (Coscioni el. al, 2018), compreendendo o nível de relações mais próximas, como por exemplo a família e a escola; 0 segundo - mesossistema - é o conjunto de microssistemas onde o ser humano se insere e frequenta de maneira ativa, sendo definido como a interação entre esses meios, por exemplo a interação da família com a escola; 0 terceiro - exossitema - envolve ambientes em que o ser humano não está diretamente, mas que Ihe causam influência direta no seu desenvolvimento, por exemplo o trabalho dos pais; e por último, 0 quarto nível do elemento contexto - macrossistema - engloba a comunidade onde a criança está inserida, sendo 
portanto o mais distante dos elementos, por exemplo, as crenças incutidas pela sociedade; 0 quinto - cronossistema - consiste em todas as modificações ambientais que ocorrem ao longo da vida e que concomitantemente influenciam o desenvolvimento e as mudanças de vida. Podem incluir alterações normais, como o início da escola, mas também podem incorporar transições de vida atípicas, como 0 divórcio dos pais (Poletto \& Koller, 2008; Antoni \& Koller, 2010; Bhering \& Sarkis, 2009; Guy-Evans, 2020).

\section{Tempo}

Permite avaliar o efeito recíproco dos elementos e termos no desenvolvimento humano ao longo da vida, 0 que se traduz por exemplo na herança familiar (Poletto \& Koller, 2008; Antoni \& Koller, 2010; Bhering \& Sarkis, 2009). Trata-se do elemento que adiciona dimensão temporal aos outros elementos que estão vinculados às pessoas, aos processos proximais e ao contexto onde ocorrem, o que permite conciliar as alterações do meio e sua estabilidade (Collodel Benetti et al. 2013). É expressado através de três noções que se unem através da perspetiva pessoal, temporal e histórica (Coscioni et al. 2018): 0 microtempo que se encontra vinculado à perspetiva pessoal, pois faz alusão ao momento exato em que os processos proximais se estabelecem; 0 mesotempo que se encontra ligado à perspetiva temporal, porque se refere à proporção e periodicidade com que se manifestam os processos proximais; e, por último, o macrotempo que corresponde à perspetiva histórica, pois assumem-se os acontecimentos que influenciam os indivíduos de forma singular e/ou coletiva (Coscioni et al. 2018). Como referiu Bronfenbrenner, o desenvolvimento do indivíduo ocorre ao longo da vida e molda-se através de contextos e circunstâncias durante o período histórico (Rosa \& Tudge, 2013), demonstrando assim a importância do elemento tempo, pois com recurso a este é possível compreender e estudar o impacto causado por diversos eventos na vida do Ser Humano (Barreto, 2016).

Partindo do argumento de Collodel Benetti e colegas (2013, p.95), "( ...) o desenvolvimento humano é (... ) interativo e contextualizado, e o indivíduo não é um ser passivo. Antes disso, é interativo, coparticipe no próprio processo de desenvolvimento, e é também dependente de outros que com ele interajam", surgindo assim 0 modelo PPCT que tem em consideração todos os seus elementos constitutivos e a interação (complexa) entre cada um deles, sendo o processo proximal o mais importante (Coscioni, et al., 2018) para a compreensão, estudo e aplicabilidade da teoria. Visando a explicação do modelo, entende-se que o mesmo está desenvolvido para se conseguir estudar o desenvolvimento humano, podendo ser aplicado ao desenvolvimento infantil, o que por si só e referindo a ideia de Antoni e Koller (2010) permite analisar os fatores de risco e de proteção e averiguar relações em meio natural de vida. A intervenção diagnóstica e preventiva estruturada sob a ótica deste modelo passa pelo ambiente escolar o que vai ao encontro da linha de pensamento de Bhering e Sarkis (2009), referindo que 0 ambiente familiar e escolar são os mais próximos do sujeito-criança e como tal, ao lidarem com a infância, abrangem aspetos diferentes da sua vida, transcendendo a aprendizagem, porque, e segundo Bronfenbrenner, "a realidade familiar, social, económica e cultural constitui um todo independente como um sistema, composto por diferentes sub-sistemas que se articulam de forma dinâmica" (Comissão Nacional de Proteção de Crianças e Jovens em Risco, 2000).

Em suma, a obra de Urie Bronfenbrenner é assinalada pela dimensão ética, "(...) acreditando ser possível tornar mais humano 0 ser humano" (Bronfenbrenner cit in Barreto, 2016, p.288), assumindo o próprio autor (Bronfenbrenner cit in Barreto, 2016) uma preocupação com gerações futuras porque se assumem sui generis, uma vez que as sociedades estão cada vez mais disformes, sendo que esta teoria poderia ser usada para melhorar as condições de vida das crianças por meio de políticas públicas (Rosa \& Tudge, 2013).

Nos dias de hoje em que as notícias nos vão revelando tantos casos de crianças fatalmente maltratadas no seio familiar, por vezes em condições economicamente bastante favoráveis, mas em que os estilo de vida e as aspirações dos adultos não permitem sobras de tempo para as crianças, temos a evidência de que houve um processo contínuo de sofrimento crescente, em que as pessoas se alhearam das necessidades básicas da criança, no seu contexto que deveria ser protetor, mas que ao longo do tempo se foi tornando cada vez mais agressor. 


\section{A IMPORTÂNCIA DA TEORIA BIOECOLÓGICA NA COMPREENSÃO E DIAGNÓSTICO dO FENÓMENO dOS MAUS TRATOS INFANTIS}

Talvez fosse o tempo de trazer para a luz do dia o modelo de Bronfenbrenner para dar resposta em várias direções às necessidades das crianças.

\section{CONCLUSÃO}

Após a análise teórica ao modelo bioecológico, percebe-se que o mesmo é usado pelas mais diversas áreas do saber, podendo ser enquadrado também à luz da ciência Criminológica, pois permite estudar e analisar comportamentos como a violência, mais especificamente os abusos infantis, uma vez que os elementos do modelo PPCT e a sua complexa interrelação permitem analisar comportamentos e com isto delinear possíveis estratégias de intervenção e prevenção de situações que se configurem de risco/perigo. Saliente-se que a relação entre os elementos constitutivos da teoria explanada considera a violência, e neste caso os maus tratos a crianças, como um resultado do desenvolvimento à luz deste paradigma. Ao estudar e analisar os elementos desta teoria, percebe-se que o meio natural de vida, associado aos contextos e pessoas que fazem parte do processo proximal, bem como 0 espaço temporal, possibilita perceber se efetivamente 0 desenvolvimento saudável da criança está em causa, visto que os processos proximais são os mais importantes e neles os pais têm um papel de destaque, acabando por tornar possível compreender se a linha que separa a salvaguarda das necessidades básicas de uma possível situação de risco/perigo se encontra fragilizada.

Em suma, a análise de cada um destes elementos permite delinear uma estratégia de prevenção e intervenção e contribuir para o correto desenvolvimento infantil, pois Bronfenbrenner também idealizou e projetou esta teoria para ser integrada em políticas públicas que auxiliassem a promoção da saúde infantil.

\section{REFERÊNCIAS BIBLIOGRÁFICAS}

Antoni, C. \& Koller, S., H. (2010). Uma família fisicamente violenta: uma visão pela teoria bioecológica do desenvolvimento humano. Temas em Psicologia 18(1), 17-30. Consultado em Maio 5, 2021, em http://pepsic.bvsalud.org/scielo.php?script=sci_arttext\&pid=\$1413-389X2010000100003.

Associação Portuguesa de Apoio à Vítima, (2011). Manual Crianças e Jovens vítimas de violência: compreender, intervir e prevenir. Consultado em Janeiro 6, 2021, em https://www.apav.pt/pdf/Manual_Criancas_Jovens_PT.pdf.

Barreto, A., C. (2016). Paradigma sistêmico no desenvolvimento humano e familiar: a teoria bioecológica de Urie Bronfenbrenner. Psicologia em Revista 22(2), 275-293. Consultado em Janeiro 6, 2021, em http://pepsic.bvsalud.org/scielo.php?script=sci_arttext\&pid=\$1677-11682016000200003.

Bhering, E. \& Sarkis, A. (2009). Modelo bioecológico do desenvolvimento de Bronfenbrenner: implicações na área da Educação Infantil. Revista Horizontes, 27(2), 7-20. Consultado em Janeiro 6, 202,1 em https://pt.scribd.com/doc/100872853/1-Modelo-bioecologico-do-desenvolvimento-de-Bronfenbrennerimplicacoes-para-as-pesquisas-na-area-da-Educacao-Infantil-16555.

Bronfenbrenner, U. \& Ceci, S. J. (1994). Nature-Nurture Reconceptualized in Developmental Perspective: A Bioecological. Psychological Review, 10(4), 568-586. Consultado em Julho 20, 2021, em https://static1.squarespace.com/static/57309137ab48de6f423b3eec/t/59aea54d4c0dbfd7d6e43622/150461 7811640/Bronfenbrenner\%26Ceci1994.pdf.

Collodel Benetti, I., Vieira, M. L., Crepaldi, M. A. \& Ribeiro Schneider, D. (2013). Fundamentos de la teoría bioecológica de Urie Bronfenbrenner. Pensando Psicología, 9(16), 89-99. Consultado em Janeiro 6, 2021, em https://www.researchgate.net/publication/305286752_Fundamentos_de_la_teoria_bioecologica_de_Urie_ Bronfenbrenner/link/5791d64d08aeb0ffcccc714d/download.

Coscioni, V., Nascimento, D. B., Rosa, E. M. \& Koller, S. H. (2018). Pressupostos teóricos-metodológicos da Teoria Bioecológica do Desenvolvimento Humano: uma pesquisa com adolescentes em medida socioeducativa. Revista Psicologia USP 29(3), 363-373. Consultado em Julho 20, 2021, em https://www.scielo.br/j/pusp/a/8NRGc4xtkbzKMWzXPvsh5RR/?format=pdf\&lang=pt. 
Comissão Nacional de Proteção de Crianças e Jovens em Risco (Coord.) (s/d). Promoção e Proteção dos Direitos das Crianças. Guia de Orientações para os Profissionais da Ação Social na Abordagem de Situações de Maus Tratos ou Outras Situações de Perigo Consultado em Julho 6, 2021, em http://ns1.adopcaointernacionalacai.gov.pt/guias/Guia_Acao_Social.pdf.

Cusson, M. (2006). Criminologia (3. ed). Alfragide: Casa das Letras.

Fonseca, A., Pinho, M. S., Simões, M. C. T. \& Simões, M. R. (2006). Psicologia Forense. Coimbra: Almedina.

Guy-Evans, 0. (2020). Bronfenbrenner's Ecological Systems Theory. [Em linha]. Simply Psychology Web site. Consultado em Maio 20, 2021, em https://www.simplypsychology.org/Bronfenbrenner.html.

Pires, A. L. D. \& Miyazaki, C. O. S. (2005). Maus tratos contra crianças e adolescentes: uma revisão da literatura para profissionais da saúde. Arq Ciênc Saúde 12(1), 42-9. Consultado em Março 6, 2021, em http://repositorio-racs.famerp.br/racs_ol/Vol-12-1/08\%20-\%20id\%20102.pdf.

Poletto, M. \& Koller, S. H. (2008). Contextos ecológicos: promotores de resiliência, fatores de risco e de proteção. Estudos de Psicologia (Campinas) 25(3), 405-416. Consultado em Janeiro 20, 2021, em https://www.scielo.br/scielo.php?pid=S0103-166X2008000300009\&script=sci_abstract\&tlng=pt.

Rosa, E. M. \& Tudge, J. (2013) Urie Bronfenbrenner s Theory of Human Development: It s Evolution from Ecology to Bioecology. Journal of Family Theory \& Review 5(4), 243-258. Consultado em Janeiro 1, 2021, em https://www.researchgate.net/publication/259552940_Urie_Bronfenbrenner's_Theory_of_Human_Develop ment_Its_Evolution_From_Ecology_to_Bioecology. 
